\title{
Cheat Sensitive Quantum Bit Commitment
}

\author{
Lucien Hardy \\ The Perimeter Institute, 35 King Street North, Waterloo, Ontario, Canada N2J 2W9 \\ Adrian Kent \\ Centre for Quantum Computation, Department of Applied Mathematics and Theoretical Physics, \\ University of Cambridge, Wilberforce Road, Cambridge CB3 0WA, United Kingdom
}

(Dated: November 1999; revised February 2004)

\begin{abstract}
We define cheat sensitive cryptographic protocols between mistrustful parties as protocols which guarantee that, if either cheats, the other has some nonzero probability of detecting the cheating. We describe an unconditionally secure cheat sensitive non-relativistic bit commitment protocol which uses quantum information to implement a task which is classically impossible; we also describe a simple relativistic protocol.
\end{abstract}

PACS numbers: 03.67.-a, 03.67.Dd, 89.70.+c

The discovery of quantum cryptography 1 and secure quantum key distribution 2] has led to much interest in understanding precisely which cryptographic tasks can be guaranteed secure by physical principles. We propose here a new class of cryptographic applications of quantum information: cheat sensitive protocols between mistrustful parties. Either party may be able to evade the intended constraints on information transfer by deviating from these protocols. However, if they do, there is a non-zero probability that the other will detect their cheating. Cheat sensitivity is potentially useful in any situation where the parties, though mistrustful, have an ongoing relationship which they value more than the potential gains from a few successful cheating attempts.

We consider here cheat sensitive protocols for bit commitment (BC), an important cryptographic primitive whose potential for physically secure implementation has been extensively investigated $2,3,4,45,6,4,6,29,10,11$, 12, 13. We first introduce BC and briefly review what is currently known about physical implementations.

Suppose $A$ and $B$ are in two different places and can send classical messages or quantum states to one another. In a classical $\mathrm{BC}$ protocol, $A$ commits herself by giving $B$ information corresponding to an encryption of a bit (either 0 or 1 ), in such a way that she can later decrypt or unveil it for him if she chooses. It should be hard (ideally, impossible) for $A$ to change the bit or for $B$ to obtain any information about the bit unless and until $A$ chooses to unveil it for him. One possible implementation is for $A$ to write the bit down on a piece of paper which she locks in a safe, and then send the locked safe to $B$. To unveil the bit later, she sends $B$ the key to the safe. One weakness of this method is that $B$ might be able to open the safe unaided, for example by picking the lock, or view its contents without opening it, for example by magnetic resonance imaging.

In general, the commitment and unveiling follow a prescribed protocol of information exchanges. We distinguish quantum protocols, which allow the exchange of quantum information, from classical protocols, which do not. We also distinguish relativistic protocols, which assume the validity of special relativity and rely on the impossibility of superluminal signalling, from nonrelativistic protocols, which do not. A BC protocol is secure, modulo certain assumptions, if it includes a parameter which can be adjusted so that the probabilities of $A$ being able to unveil a state significantly different from the committed state and of $B$ being able to extract significant information can simultaneously be made arbitrarily small. It is unconditionally secure within a given physical theory if the only assumption necessary is the validity of that theory. (Formal security definitions can be found in, e.g., Refs. [6, 13].)

Several quantum BC schemes have been proposed [2, 3, 4, 14]. These schemes presently offer good practical security, but in principle are insecure. Indeed, LoChau [5, 8] and Mayers [6, 7, 9] showed that no nonrelativistic quantum BC schemes can be perfectly secure against both parties. Mayers [6, 7, 9] extended this to prove the impossibility of unconditionally secure nonrelativistic quantum $\mathrm{BC}$ and to cover models in which classical and quantum information are treated separately. Unconditionally secure classical relativistic BC protocols exist [1, 12]. It is conjectured that these protocols are also secure against quantum attack.

The essential weakness in non-relativistic quantum BC protocols highlighted by Lo-Chau and Mayers is that, whenever $B$ can extract no (or little) information about the committed bit, $A$ can (or very probably can) undetectably alter the commitment from 0 to 1 or vice versa.

A separate issue in quantum protocols is that $A$ may commit an improper mixture of bit states 10, 13, in which the committed bit is entangled with another state. This is not advantageous when BCs are used in isolation - for example, to record a secret prediction — but can be when they are used as subprotocols for a larger task. A BC protocol which forces $A$ to commit a fixed classical bit, 0 or 1 , is said to implement bit commitment with a certificate of classicality (BCCC). Secure BCCC protocols based on reasonable quantum computational complexity assumptions might be possible 14,15$]$, but no unconditionally secure BCCC protocols exist 13$]$. 
Relativistic BC schemes 11, 12] offer the prospect of practical unconditional security. However, they require the maintenance of separated sites and the continual use of communication channels between commitment and unveiling. For some applications these constraints may be serious disadvantages. Hence, as unconditionally secure non-relativistic BC is impossible, it is interesting to explore what can be achieved by non-relativistic protocols. We show here that cheat sensitive $\mathrm{BC}$ can be implemented non-relativistically. We also give a simple relativistic cheat sensitive BC protocol.

Defining cheat sensitivity A cheat sensitive quantum $\mathrm{BC}$ protocol is a quantum $\mathrm{BC}$ protocol in which, assuming that the commitment will eventually be unveiled, $A$ cannot alter the probabilities of her revealing a 0 or 1 after the commitment without risking detection and $B$ cannot extract information about the committed bit before the unveiling without risking detection. Note that by this definition the detection probabilities only need be nonzero to imply cheat sensitivity.

Protocols If a BC is encoded by non-orthogonal quantum states, $B$ cannot extract information without disturbing the states. This means he risks detection if he later has to return the committed state. At the same time, $A$ risks detection if she sends one state and later tries to claim that she sent the other. This suggests a strategy for cheat sensitive BC. The problem is to arrange for both parties to be simultaneously at risk of cheat detection. Standard quantum BC methods do not work here. For example, a protocol can not be cheat sensitive if $A$ tells $B$ at revelation what the committed state was, since $B$ can then return a copy even if he has disturbed the original. As we shall show, there are ways around this difficulty.

We now describe two cheat sensitive quantum BC protocols. We take $|0\rangle,|1\rangle$ as orthonormal qubit states and write $| \pm\rangle=\frac{1}{\sqrt{2}}(|0\rangle \pm|1\rangle)$.

Protocol 1: non-relativistic CSBC

Stage 0: the prelude. $B$ prepares a singlet state, $\left|\Psi^{-}\right\rangle_{A B}=\frac{1}{\sqrt{2}}\left(|0\rangle_{A}|1\rangle_{B}-|1\rangle_{A}|0\rangle_{B}\right)$, and sends qubit $A$ to $A$. At certain stages of the protocol either party may "challenge" this singlet. This means that the other party must send a qubit which is supposed to be their half of the singlet to the challenger, who can then check that the two qubit state is indeed a singlet by measuring the relevant projection. If not, there is a non-zero probability it will fail the test.

Stage 1: the commitment. The protocol allows a simple commitment procedure which $A$ may use if she wishes to commit to a definite classical bit: to commit to 0 , she prepares a qubit $C$ chosen randomly to be either $|0\rangle$ or $|-\rangle$, each with probability $1 / 2$; to commit to 1 , she similarly prepares either $|1\rangle$ or $|+\rangle$. Then she sends the $C$ qubit to $B$.

As usual in quantum BC protocols, $A$ is allowed a more general non-classical commitment, in which she instead prepares a state $|\psi\rangle=\sum_{r=0,1,+,-}\left|\alpha_{r}\right\rangle_{A}|r\rangle_{C}$, where the unnormalised states $\left|\alpha_{r}\right\rangle$ are orthogonal, keeps the |\rangle$_{A}$ system under her control, and sends the $|C\rangle$ qubit to $B$. We will show later that the probabilities for her unveiling the classical bit are fixed by such a commitment, in the sense that they cannot subsequently be altered without cheating and risking detection.

Stage 2: the unveiling. $A$ is first given the option of challenging the singlet. If she does and it fails the test, she has detected cheating. Next, whether or not she made a challenge, she must reveal the value of the committed classical bit (but not the qubit used to encode it). $B$ then has the option of challenging the singlet, if $A$ did not. If he does and it fails the test, he has detected cheating.

Stage 3: the game. If either party earlier challenged the singlet, they automatically lose the game. If neither challenged the singlet, they now each measure their singlet qubit in the $|0\rangle,|1\rangle$ basis. $B$ sends his result to $A$. If hers is not opposite, she has detected cheating. If $B$ reports the result 1 then $A$ loses the game; if 0 then $B$ loses.

If $A$ loses she must reveal which state was used to encode the committed bit in the qubit $C$. $B$ then measures $C$ to check it is in the state $A$ claims. If not, he has detected cheating.

If $B$ loses he must return the qubit $C$ to $A$. She makes a measurement to check it is still in the state she originally prepared. If not, she has detected cheating.

This completes the protocol. Note that the cheating tests detect only that someone - possibly the party carrying out the test - has cheated. The ambiguity here is not a worry since, as usual in mistrustful cryptography, the protocol is designed to protect honest parties against cheats, not necessarily to protect one cheat against another.

A party might choose to terminate early if they detect the other cheating. However, we have not stipulated this, since they might choose to continue if that seems advantageous.

Proof of cheat-sensitivity: The security proof relies on the following facts: 1) $B$ cannot send anything other than a half-singlet without risking failing a singlet challenge. 2) If A or B carry out any non-trivial measurement on the singlet they risk failing a singlet challenge. If A preemptively makes her own challenge to avoid being challenged, she ensures she will lose the game, and this forces her to make an honest commitment and unveiling. 3) When A and B can no longer be challenged, they cannot advantageously use their singlet qubit in any quantum information processing.

As we will show, these facts imply that the singlet can be effectively factored out from the rest of the protocol and merely acts to provide a random "loser" in the game. Neither party can be certain they will not lose the game. This prevents them from cheating: $A$ may have to tell $B$ what state the qubit $C$ is in when this qubit is in $B$ 's hands, and $B$ may have to return the qubit $C$ to $A$ in its original state.

Clearly, at any stage, $A$ and $B$ can apply a reversible local unitary operation to the quantum states under their 
control without fear of detection. We take this as understood below, rather than repeating the phrase "up to a local unitary operation" at each stage, since applying a local unitary does not per se gain a cheat anything.

To begin the proof, note that $B$ must prepare a singlet and send half of it to $A$, since $A$ may challenge the singlet. The following lemma shows that once $A$ and $B$ share the singlet neither of them can carry out nontrivial quantum operations on it.

Lemma 1. Suppose that A and B share a state $\left|\Psi^{-}\right\rangle_{A B}|\psi\rangle_{A B}$, where $\left|\Psi^{-}\right\rangle_{A B} \in H_{a}^{2} \otimes H_{b}^{2}$ and $|\psi\rangle_{A B} \in$ $H_{a}^{m} \otimes H_{b}^{n}$, so that A's subsystem lies in $H_{A}=H_{a}^{2} \otimes H_{a}^{m}$ and B's in $H_{B}=H_{b}^{2} \otimes H_{b}^{m}$. Here $H_{a}^{d}$ and $H_{b}^{d}$ denote $d$ dimensional quantum systems initially under $A$ 's and $B$ 's control respectively. Suppose A applies a quantum measurement, defined by Kraus operators $E_{i}$ corresponding to outcomes $i$, on $H_{A}$ and then returns the $H_{a}^{2}$ qubit to $\mathrm{B}$. If it is the case that, for all values of the measurement outcome $i$, B now possesses the singlet $\left|\Psi^{-}\right\rangle$in $H_{a}^{2} \otimes H_{b}^{2}$, then the Kraus operators $E_{i}$ must take the form $I \otimes E_{i}^{\prime}$, where the $E_{i}^{\prime}$ define Kraus operators for a quantum operation on $H_{a}^{m}$.

Proof. $\quad E_{i} \otimes I_{B}\left|\Psi^{-}\right\rangle_{A B}|\psi\rangle_{A B}=\left|\Psi^{-}\right\rangle_{A B}\left|\psi_{i}\right\rangle_{A B}$. As $E_{i} \otimes I_{B}|0\rangle_{a}|1\rangle_{b}|\psi\rangle_{A B}=|0\rangle_{a}|1\rangle_{b}\left|\psi_{i}\right\rangle_{A B}$ and $E_{i} \otimes$ $I_{B}|1\rangle_{a}|0\rangle_{b}|\psi\rangle_{A B}=|1\rangle_{a}|0\rangle_{b}\left|\psi_{i}\right\rangle_{A B}$, linearity implies that $E_{i}$ acts as the identity on $H_{a}^{2}$. QED.

This implies that, unless $A$ challenges the singlet herself before revealing her classical bit, any strategy by which she generates the classical bit value sent to $B$ cannot involve nontrivial operations on her singlet qubit. Similarly, any strategy by which $B$ extracts classical information before $A$ 's unveiling cannot involve non-trivial operations on his singlet qubit.

Now consider an honest $A$ and dishonest $B$. For $B$ to cheat successfully, he must extract some information about the committed bit before the unveiling. We have established that he cannot perform any operations on his singlet qubit before the classical bit is unveiled. Thus he must restrict his attention to qubit $C$ up to this point. $B$ would like to know, before the unveiling begins, whether the committed state is $|0\rangle$ or $|-\rangle$ corresponding to a 0 or $|1\rangle$ or $|+\rangle$ corresponding to a 1 . The most general way he can extract information is to introduce an ancilla $|P\rangle$, apply a unitary operation $U_{1}$, and then measure part of the system, creating the state

$$
U_{1}|P\rangle|r\rangle_{C}=\sum_{i} c_{r}^{i}|i\rangle\left|\eta_{r}^{i}\right\rangle
$$

where $r=0,1,+,-. \quad B$ measures onto the states $|i\rangle$ (which are orthonormal). For outcome $i$ he will possess the state $\left|\eta_{r}^{i}\right\rangle$. When $A$ declares the committed bit in step 1 of the unveiling $B$ will know he has one of two states. However, there is no deterministic algorithm for decreasing the overlap between two states.

We first assume $B$ makes no nontrivial use of his singlet qubit after $A$ 's unveiling, $B$ must thus ensure that

$$
\left|\left\langle\eta_{0}^{i} \mid \eta_{-}^{i}\right\rangle\right| \leq|\langle 0 \mid-\rangle| \quad \text { and } \quad\left|\left\langle\eta_{1}^{i} \mid \eta_{+}^{i}\right\rangle\right| \leq|\langle 1 \mid+\rangle|
$$

so he can send the correct state to $A$ in stage 3 if he loses the game. We will now see that under these conditions $B$ can extract no information about the commitment before unveiling. Consider applying the controlled unitary operator $U_{2}=\sum_{i}|i\rangle\langle i| \otimes U^{i}$ to the RHS of (11), where

$$
U^{i}\left|\eta_{0}^{i}\right\rangle=|0\rangle, \quad U^{i}\left|\eta_{-}^{i}\right\rangle=a^{i}|0\rangle-b^{i}|1\rangle,
$$

and without loss of generality (redefining $\left|\eta_{-}^{i}\right\rangle$ by a phase factor if necessary) we take $a^{i}$ and $b^{i}$ to be real and positive. From (2) it follows that $a^{i} \leq \frac{1}{\sqrt{2}} \leq b^{i}$. Let $U=U_{2} U_{1}$. We have

$$
\begin{aligned}
U|P\rangle|0\rangle & =\left(\sum_{i} c_{0}^{i}|i\rangle\right)|0\rangle \\
U|P\rangle|-\rangle & =\left(\sum_{i} c_{-}^{i} a^{i}|i\rangle\right)|0\rangle-\left(\sum_{i} c_{-}^{i} b^{i}|i\rangle\right)|1\rangle .
\end{aligned}
$$

However, we also have

$$
U|P\rangle|-\rangle=U \frac{1}{\sqrt{2}}|P\rangle(|0\rangle-|1\rangle)=\frac{1}{\sqrt{2}}\left(\sum_{i} c_{0}^{i}|i\rangle\right)|0\rangle+|\perp\rangle
$$

where $|\perp\rangle$ is orthogonal to the first term on the RHS of (4). We can put $|\perp\rangle=\alpha|A\rangle|0\rangle+\beta|B\rangle|1\rangle$ where $|\alpha|^{2}+|\beta|^{2}=\frac{1}{2}$. Comparing (3) and (4) we obtain $|\beta|^{2}=\sum_{i}\left|c_{-}^{i} b^{i}\right|^{2} \leq \frac{1}{2} \leq \sum_{i}\left|c_{-}^{i} a^{i}\right|^{2}$. Since $a^{i} \leq b^{i}$, this implies that $a^{i}=b^{i}=\frac{1}{\sqrt{2}}$ for all $i$ for which $c_{-}^{i} \neq 0$. Hence, comparing (3) and (4) (and since we now have $\alpha=0$ ) we obtain $c_{-}^{i}=c_{0}^{i}$ for all $i$. Also,

$$
\begin{aligned}
& U|P\rangle|1\rangle=U|P\rangle(|0\rangle-\sqrt{2}|-\rangle) \\
& \quad=\sum_{i} c_{0}^{i}|i\rangle(|0\rangle-\sqrt{2}|-\rangle)=\sum_{i} c_{0}^{i}|i\rangle|1\rangle
\end{aligned}
$$

Hence, $\left|c_{1}^{i}\right|=\left|c_{0}^{i}\right|$ for all $i$, and similarly $\left|c_{+}^{i}\right|=\left|c_{-}^{i}\right|$ for all $i$. Thus the probability of a given outcome $i$ cannot depend on the bit committed, and so no information about that bit can be extracted without $B$ risking detection.

Now we need to exclude the possibility of $B$ cheating without risk of detection by extracting classical information from $A$ 's commitment qubit and then carrying out some nontrivial operation on that qubit and his singlet qubit between the unveiling and the game. The most general state $A$ and $B$ may share just after unveiling is $\left|\Psi^{-}\right\rangle_{A B}\left(\left|\alpha_{1}\right\rangle_{A}\left|\psi_{1}\right\rangle_{B}+\left|\alpha_{2}\right\rangle_{A}\left|\psi_{2}\right\rangle_{B}\right)$, where the first state is the as yet undisturbed singlet and the second is a state entangling two of $A$ 's orthogonal control states $\left|\alpha_{i}\right\rangle$ with two normalised states $\left|\psi_{i}\right\rangle$ resulting from $B$ 's actions on the two states (say $|0\rangle,|-\rangle$ ) corresponding to $A$ 's unveiling c-bit. If $B$ has extracted useful information, we have $\left|\left\langle\psi_{1} \mid \psi_{2}\right\rangle\right|>|\langle 0 \mid-\rangle| . B$ must now generate a bit to tell $A$ whether or not he will challenge, and without loss of generality we can assume he does so by applying a local unitary operation to create a qubit |\rangle$_{c}$ which is sent to $A$, where $|0\rangle_{c}$ and $|1\rangle_{c}$ declare respectively no challenge and a challenge. Considering the constraints on $B$ implied by the protocol, given that he wishes to avoid any risk of 
cheating detectably, we see the unitary operation must implement a map of the form:

$$
\begin{array}{r}
\left|\Psi^{-}\right\rangle_{A B}\left(\left|\alpha_{1}\right\rangle_{A}\left|\psi_{1}\right\rangle_{B}+\left|\alpha_{2}\right\rangle_{A}\left|\psi_{2}\right\rangle_{B}\right)\left|*_{0}\right\rangle_{c} \rightarrow \\
\left|*_{1}\right\rangle_{A B}\left(\left|\alpha_{1}\right\rangle_{A}|0\rangle_{B}+\left|\alpha_{2}\right\rangle_{A}|-\rangle_{B}\right)|1\rangle_{c} \\
+|0\rangle_{A}|1\rangle_{B}\left|*_{2}\right\rangle_{A B}|0\rangle_{C} \\
+|1\rangle_{A}|0\rangle_{B}\left(\left|\alpha_{1}\right\rangle_{A}|0\rangle_{B}+\left|\alpha_{2}\right\rangle_{A}|-\rangle_{B}\right)|0\rangle_{c}
\end{array}
$$

where $\left|*_{i}\right\rangle$ are unspecified states. Considering the components of $|1\rangle_{A}\left|\alpha_{i}\right\rangle_{A}$ in this equation, we see that in particular the unitary operation must deterministically map states with overlap $\left|\left\langle\psi_{1} \mid \psi_{2}\right\rangle\right|$ to states with overlap no greater than $|\langle 0 \mid-\rangle|$. That is, it must deterministically decrease the overlap, which is impossible.

Now consider an honest $B$ and a dishonest $A$. The most general thing $A$ can do initially is entangle the qubit $C$ with some ancilla $A$ which she keeps. Then the entangled state she prepares can be written as $|\psi\rangle=$ $\sum_{r=0,1,+,-}\left|\alpha_{r}\right\rangle_{A}|r\rangle_{C}$. The states $\left|\alpha_{r}\right\rangle_{A}$ need not be normalized, nor do we impose at this stage that they are orthogonal. Further, since the states $|r\rangle$ form an overcomplete basis, the states $\left|\alpha_{r}\right\rangle$ need not be unique. We will show that, if $A$ is to be certain of not being caught cheating, then there must be some way of writing the expansion above such that states $\left|\alpha_{r}\right\rangle$ are indeed orthogonal (if they have non-zero norm). To see this consider what happens at the unveiling stage. $A$ must announce the committed classical bit to $B$. The most general thing she can do is perform a unitary operation on $A$ of the form

$$
\left|\alpha_{r}\right\rangle_{A} \longrightarrow \sum_{k=0,1}\left|\beta_{r k}\right\rangle_{A^{\prime}}|k\rangle_{A^{\prime \prime}}
$$

and measure on $A^{\prime \prime}$ to extract a bit, $k=0,1$, which she sends to $B$ as the committed classical bit. At this stage $A$ may have already challenged the singlet in which case she has lost the game and must tell $B$ what the state of the qubit $C$ is. Alternatively, she may not have challenged the singlet. Then $B$ may challenge the singlet. If he declines he can immediately measure his singlet qubit in the $|0\rangle,|1\rangle$ basis. He may get a 1 in which case $A$ loses the game and must tell $B$ what the state of qubit $C$ is. $A$ 's singlet qubit will, in this case, be collapsed into a definite pure state and, hence, is of no use to $A$ in any cheat strategy. In the case where $A$ gets $k=0$ she must be able to collapse the qubit $C$ onto either $|0\rangle$ or $|-\rangle$ by making a measurement on $A^{\prime}$ and hence we must have

$$
\sum_{r=0,1,+,-}\left|\beta_{r 0}\right\rangle|r\rangle=|u\rangle|0\rangle+\left|u^{\perp}\right\rangle|-\rangle
$$

where $\left\langle u \mid u^{\perp}\right\rangle=0$ and, similarly, for the $k=1$ case, we must have

$$
\sum_{r=0,1,+,-}\left|\beta_{r 1}\right\rangle|r\rangle=|v\rangle|0\rangle+\left|v^{\perp}\right\rangle|-\rangle
$$

where $\left\langle v \mid v^{\perp}\right\rangle=0$. This means that we can write $\widehat{U}_{A} \otimes$ $\widehat{I}_{C}|\psi\rangle=|\tilde{1}\rangle_{A}|0\rangle_{C}+|\tilde{2}\rangle_{A}|-\rangle_{C}+|\tilde{3}\rangle_{A}|1\rangle_{C}+|\tilde{4}\rangle_{A}|+\rangle_{C}$, where
$|\tilde{1}\rangle_{A}=|u\rangle_{A^{\prime}}|0\rangle_{A^{\prime \prime}}$, etc., and $\widehat{U}_{A}$ is the unitary operator mentioned above that $A$ may apply at the unveiling stage. The states $|\tilde{i}\rangle$ are orthogonal. The probabilities that $A$ declares a 0,1 are given by

$$
p_{0}=\langle\tilde{1} \mid \tilde{1}\rangle+\langle\tilde{2} \mid \tilde{2}\rangle, \quad p_{1}=1-p_{0},
$$

As the states $|\tilde{i}\rangle$ are orthogonal, we have

$$
p_{0}=\operatorname{Tr}\left(\rho_{C} \sigma_{C}\right)-\frac{1}{2}, \quad p_{1}=1-p_{0},
$$

where $\rho_{C}=\operatorname{Tr}_{A}(|\psi\rangle\langle\psi|)$ and $\sigma_{C}=|0\rangle_{C}\left\langle\left. 0\right|_{C}+\mid-\right\rangle_{C}\left\langle-\left.\right|_{C}\right.$. Since these probabilities only depend on the reduced density matrix of the qubit $C$ which is in $B$ 's hands, there is nothing $A$ can do to alter them once she has sent this qubit to $B$ at the commitment stage. Hence we see that, if she is to be certain of avoiding being detected cheating, she cannot alter the probabilities of declaring a 0 or a 1 .

Protocol 2: relativistic $C S B C$ We now describe a simple relativistic cheat sensitive $\mathrm{BC}$ protocol. $A$ and $B$ agree two non-orthogonal commitment states, $\left|\psi_{0}\right\rangle$ and $\left|\psi_{1}\right\rangle$, corresponding to commitments of 0 and 1 . $A$ sends $B$ the state corresponding to her commitment. When $A$ is ready to unveil, she and $B$ set up two extra separated sites, $A_{1}$ and $B_{1}$ relatively near the main sites $A$ and $B$ which they occupy, and $A_{2}$ and $B_{2}$ further away, with the separations such that $d(A, B) \approx d\left(A_{1}, B_{1}\right) \approx$ $d\left(A_{2}, B_{2}\right)<<d\left(A, A_{1}\right)<<d\left(A, A_{2}\right)$. Each party can verify these separations by timing the receipt of messages, so no trust is required here 16$]. A_{2}$ then reveals the committed bit to $B_{2}$. Before this information can reach $A, B, A_{1}$ and $B_{1}$, these four carry out a relativistic coin tossing protocol 16$]$. If they obtain a $0, B$ returns the commitment state to $A$ for testing; if a $1, B$ keeps it and tests it once $B_{2}$ has informed him of $A_{2}$ 's revelation.

Discussion Quantum information allows us to implement unconditionally secure cheat sensitive BC by relatively simple protocols, which will easily be implementable when the technology for quantum state storage is developed. The mechanism for cheat sensitivity used in these protocols relies on the properties of quantum information: classical information cannot be used in the same way, since $A$ cannot be sure if $B$ has extracted information from a classical message. It would be interesting to understand how to optimise the levels of cheat sensitivity against $A$ and $B$, and to quantify the effect of noise (which would tend to make our protocols imperfectly cheat sensitive).

Finally, our results suggest the possibility of cheat sensitive implementations of other cryptographic tasks, such as non-relativistic quantum multi-party computation for general functions, for which unconditional security is not always attainable 17]. It would be interesting to understand precisely which tasks can be implemented with cheat sensitivity.

After submitting the first version of this paper, we learned of independent work by Aharonov et al. [18], who define and implement a related weaker cryptographic task, quantum bit escrow. 


\section{Acknowledgments}

We thank Dorit Aharonov and Amnon Ta-Shma for careful criticisms of earlier protocols and Jeffrey Bub, Daniel Gottesman, Hoi-Kwong Lo, Dominic Mayers and Rob Spekkens for helpful comments. We acknowledge support from Royal Society University Research Fellow- ships at the Universities of Oxford and Cambridge and partial support from an HP Bursary and the EU project PROSECCO. AK thanks the Perimeter Institute for hospitality.
[1] S. Wiesner, SIGACT News 15 (1983) 78.

[2] C.H. Bennett and G. Brassard, in Proceedings of IEEE International Conference on Computers, Systems and Signal Processing (IEEE, New York, 1984), p. 175.

[3] G. Brassard et al., in Proceedings of the 34th Annual IEEE Symposium on the Foundation of Computer Science (IEEE Comp. Soc., Los Alamitos, California, 1993), p. 362 .

[4] G. Brassard and C. Crépeau, in Advances in Cryptology: Proceedings of Crypto'90, Lecture Notes in Computer Science Vol 537 (Springer-Verlag, Berlin, 1991), p. 49.

[5] H.-K. Lo and H. Chau, Phys. Rev. Lett. 78 (1997) 3410.

[6] D. Mayers, Phys. Rev. Lett. 78 (1997) 3414.

[7] D. Mayers, quant-ph/9603015

[8] H.-K. Lo and H. Chau, Physica D 120 (1998) 177.

[9] D. Mayers, in Proceedings of the Fourth Workshop on
Physics and Computation (New England Complex System Inst., Boston, 1996), p. 226.

[10] G. Brassard et al., quant-ph/9806031

[11] A. Kent, Phys. Rev. Lett. 83 (1999) 1447-1450.

[12] A. Kent, quant-ph/9906103 to appear in J. Cryptology.

[13] A. Kent, Phys. Rev. A 61, 042301 (2000).

[14] L. Salvail, in Proceedings of Crypto'98, Lecture Notes in Computer Science Vol 1462 (Springer-Verlag, SantaBarbara, 1998) pp. 338-353.

[15] A. Kent, Phys. Rev. A 68 (2003) 012312.

[16] A. Kent, Phys. Rev. Lett. 83 (1999) 5382-5384.

[17] H.-K. Lo, Phys. Rev. A 56 (1997) 1154.

[18] D. Aharonov et al., in Proceedings of the 32nd Annual ACM Symposium on the Theory of Computing (New York, 2000), ACM Press, pp. 705-714. quant-ph/0004017 